\title{
The effect of osteoprotegerin on implant osseointegration in ovariectomized rats
}

\author{
Yiming Liu, Jing Hu, Biao Liu, Xiliang Jiang, Yunfeng Li
}

State Key Laboratory of Oral Diseases, National Clinical Research Center for Oral Diseases, Department of Oral and Maxillofacial Surgery, West China Hospital of Stomatology, Sichuan University, Chengdu, China

Submitted: 27 February 2015

Accepted: 12 March 2015

Arch Med Sci 2017; 13, 2: 489-495

DOI: https://doi.org/10.5114/aoms.2017.65468

Copyright $\odot 2017$ Termedia \& Banach

\section{Abstract}

Introduction: Osteoprotegerin (OPG), the endogenous inhibitor of RANKL, prevents or reverses bone loss in a variety of preclinical models of bone disease. Preclinical studies indicate that osteoporosis significantly impairs implant fixation. This study aims to investigate the role of OPG in implant osseointegration in ovariectomized rats.

Material and methods: Twelve weeks after bilateral ovariectomy, each rat accepted two titanium screws in the proximal tibiae. All animals were then randomly divided into two groups: the control (10 rats) and OPG group (10 rats). Subcutaneous injection of OPG $(10 \mathrm{mg} / \mathrm{kg})$ or vehicle was performed three times a week. Eight weeks later, tibiae with screws were harvested for micro-computed tomography $(\mu \mathrm{CT})$, histological and biomechanical analysis. Results: Compared to control, OPG increased the percent bone volume by $124 \%$, the percent osseointegration by $167 \%$, the mean trabecular number by $111 \%$, the mean trabecular thickness by $92 \%(p<0.01)$, the mean connective density by $95 \%(p<0.05)$; and decreased the mean trabecular separation by $64 \%$ in $\mu \mathrm{CT}$ analysis $(p<0.05)$. Osteoprotegerin also increased bone area density by $160 \%$ and bone-to-implant contact by $234 \%$ in histomorphometric evaluation $(p<0.01)$, and increased the maximal push-out force by $228 \%$ in biomechanical test $(p<0.01)$.

Conclusions: Systemic administration of OPG improved implant osseointegration and fixation in ovariectomized rats, resulting from the increased peri-implant bone mass and improved trabecular microarchitecture.

Key words: implant, osseointegration, osteoporosis, osteoprotegerin, rat.

\section{Introduction}

Osteoporosis is a common skeletal disease with serious clinical consequences due to an increased bone turnover rate, characterized by low bone mass, deteriorated bone microstructure, and increased risk of fracture [1]. With the increased aging of the population, the number of patients affected by osteoporosis is also increasing [2, 3]. Studies on animals indicated that osteoporosis significantly impaired implant osseointegration and fixation $[4,5]$. Results from clinical studies showed that osseointegration of dental implants might be obtained in osteoporotic bone, but a longer time should be considered [6-8]. Information from orthopedic procedures indicated that osteoporosis seemed to exert a more severe influence on joint arthroplasty than a dental implant, due to the differing effect of osteoporosis on long bones and jaw bones [9-11].
Corresponding author:

Yunfeng Li

Sichuan University

No. 14 , Section 3

South Renmin Road

610041 Chengdu, China

Phone: +86 2885502334

E-mail: doctorlyf@163.com 
Since osteoporosis is characterized by increased bone resorption and deficient bone formation, anti-catabolic and anabolic agents have been investigated to improve implant fixation in osteoporotic bone. Bisphosphonates (BPs) are a class of agents that effectively inhibit osteoclast-mediated bone resorption, but also inhibit bone formation due to the network of osteoclasts and osteoblasts [12, 13]. Parathyroid hormone (PTH), a representative of anabolic drugs, enhances implant fixation by increasing bone turnover, with greater effects on bone formation than bone resorption, but the withdrawal of PTH was reported to cause deterioration of implant fixation in rats [14].

Osteoclasts play a crucial role in the occurrence of osteoporosis due to imbalance of bone resorption and formation. The receptor activator of nuclear factor- $\kappa B$ ligand (RANKL), a cytokine member of the tumor necrosis factor family, is up to now the most important factor that induces osteoclastogenesis [15]. Inhibition of RANKL by denosumab is suggested to reduce the rate of bone remodeling more than alendronate $[16,17]$. Osteoporotic patients frequently accepted implant treatment due to a denture defect or joint problems. Thus, it is important to know the effect of inhibition of RANKL on implant fixation in osteoporotic bone. However, information about the role of denosumab on bone healing in animals is limited, since denosumab does not recognize murine RANKL and has no apparent effects in mice or rats [18].

Osteoprotegerin (OPG), a decoy receptor, is the endogenous inhibitor of RANKL, and OPG treatment prevents or reverses bone loss in a variety of preclinical models of bone disease [19]. The purpose of this study is to evaluate the effect of inhibition of RANKL by OPG on implant osseointegration in the proximal tibiae of ovariectomized (OVX) rats. After 8-week treatment with

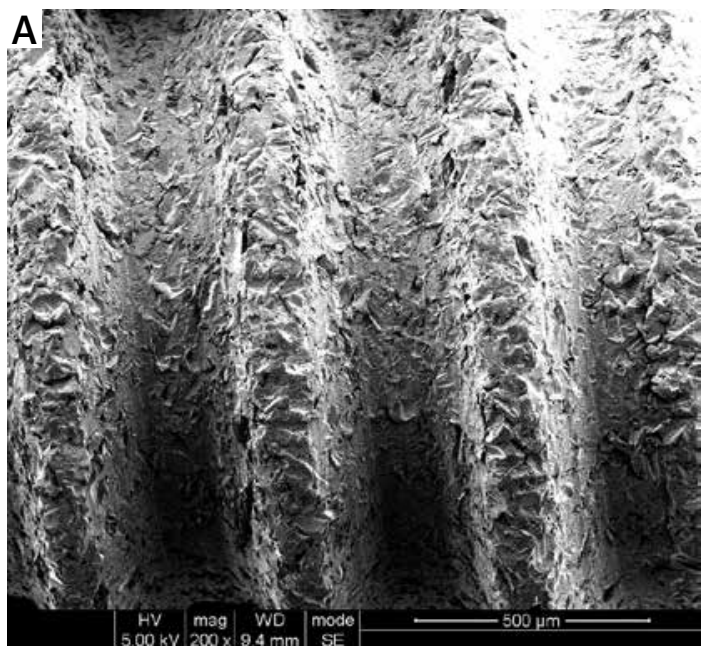

OPG or vehicle, tibiae with titanium screws were harvested for $\mu \mathrm{CT}$, histological and biomechanical analysis.

\section{Material and methods}

\section{Animals}

Twenty 3-month-old female Sprague-Dawley rats were included in this study. Each group of 4 animals was kept in one cage under climate-controlled conditions $\left(25^{\circ} \mathrm{C} ; 55 \%\right.$ humidity; $12 \mathrm{~h}$ of light alternating with $12 \mathrm{~h}$ of darkness), supplied with the standard laboratory diet and tap water. The experimental protocol was approved by the Ethics in Animal Research Committee of Sichuan University.

\section{Implants}

Titanium screws with $1.8-\mathrm{mm}$ outer diameter, 3.5- $\mathrm{mm}$ length, and $0.5-\mathrm{mm}$ pitch were used in this study (National Engineering Research Center for Biomaterials, Sichuan University). All screws were grit-blasted with $25 \mu \mathrm{m}$ aluminium oxide $\left(\mathrm{Al}_{2} \mathrm{O}_{3}\right)$ particles. Figure 1 shows the surface morphology of the screw by scanning electron microscopy (SEM).

\section{Surgical procedures}

After 1-week acclimatization, bilateral ovariectomy was performed on all animals. Twelve weeks later, each OVX rat received two implants in the proximal tibiae bilaterally as previously described [20]. General anesthesia was obtained by abdominal injections of ketamine $(100 \mathrm{mg} / \mathrm{kg}$; 3B Scientific Corporation, USA) and xylazine (10 mg/ kg; Atomax Chemicals Co., Ltd., China). All procedures were done under aseptic conditions. Briefly, a $1.6-\mathrm{mm}$ hole located $3 \mathrm{~mm}$ distal to the proximal growth plate was drilled, then implants were

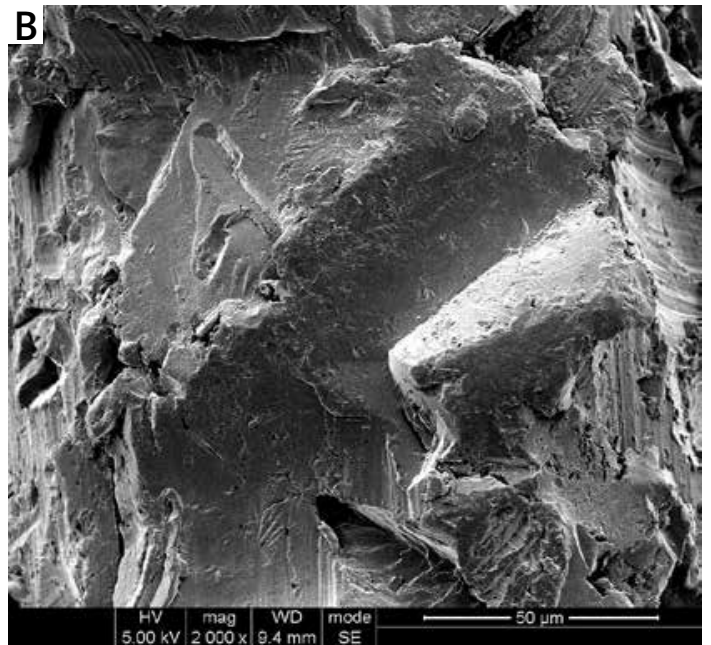

Figure 1. Scanning electron microscope (SEM) micrographs of the surface topography of screws at different magnifications: A - 200x, B - 2000x 
screwed in with gentle force [21]. All animals received intramuscular antibiotic and analgesic injection for 3 postoperative days.

\section{Pharmaceutical treatment}

Pharmaceutical intervention started on the first post-operative day. All rats randomly accepted subcutaneous injection of recombinant rat PEG-OPG (10 mg/kg, Amgen Inc; Thousand Oaks, CA, USA) or vehicle three times a week, with ten rats in each group. The dose of OPG was defined according to a previous animal study [22]. After 8-week treatment, all animals were killed by injection of overdosed pentobarbital in the heart under general anesthesia, and specimens with screws were harvested for $\mu \mathrm{CT}$, histomorphometry, and biomechanical analysis.

\section{$\mu \mathrm{CT}$ analysis}

The right tibiae with screws ( $n=10$ /group) were scanned on a $\mu \mathrm{CT}$ system (70 kV, $114 \mu \mathrm{A} ; \mu$-CT 80 scanner Scanco Medical, Bassersdorf, Switzerland). The volume of interest (VOI) included the trabecular compartment extending $1150 \mu \mathrm{m}$ from the longitudinal axis of the screw, namely 250$500 \mu \mathrm{m}$ from the surface of screws (Figure $2 \mathrm{~A}$ ). The VOI was chosen equally long for all samples, including the entire length of the threaded part of screws, but the cortical bone was excluded semi-automatically if within VOI. The $\mu \mathrm{CT}$ pictures were taken with a resolution of $2048 \times 2048$ pixels and an isotropic voxel size of $10 \mu \mathrm{m}$. The percent bone volume (BV/TV), mean trabecular thickness (Tb.Th), mean trabecular number (Tb.N), mean trabecular separation (Tb.Sp), and mean connective density (Conn.D) were quantitatively analyzed within the VOI. The percent osseointegration (\%OI) was calculated as the ratio between bone and total voxels in direct contact with the implant in cancellous bone.

\section{Histological evaluation}

After the $\mu \mathrm{CT}$ scan, the right tibiae with screws were prepared for undecalcified histological sections. Transverse sections through the long axis of screws and perpendicular to the long axis of tibiae were prepared using a rotary diamond saw (SP1600/2600, Leica, Germany) and stained in 1\% toluidine blue. Bone-to-implant contact ratio (BIC) and bone area density (BA) were assessed using Leica DMI 6000B micro-systems (Germany). The region of interest (ROI) was defined on sections similar to VOI in $\mu \mathrm{CT}$ analysis, but restricted to the two front most threads (Figure $2 \mathrm{~B}$ ).

\section{Biomechanical test}

The left tibiae with screws were tested on a commercial material testing system (Instron
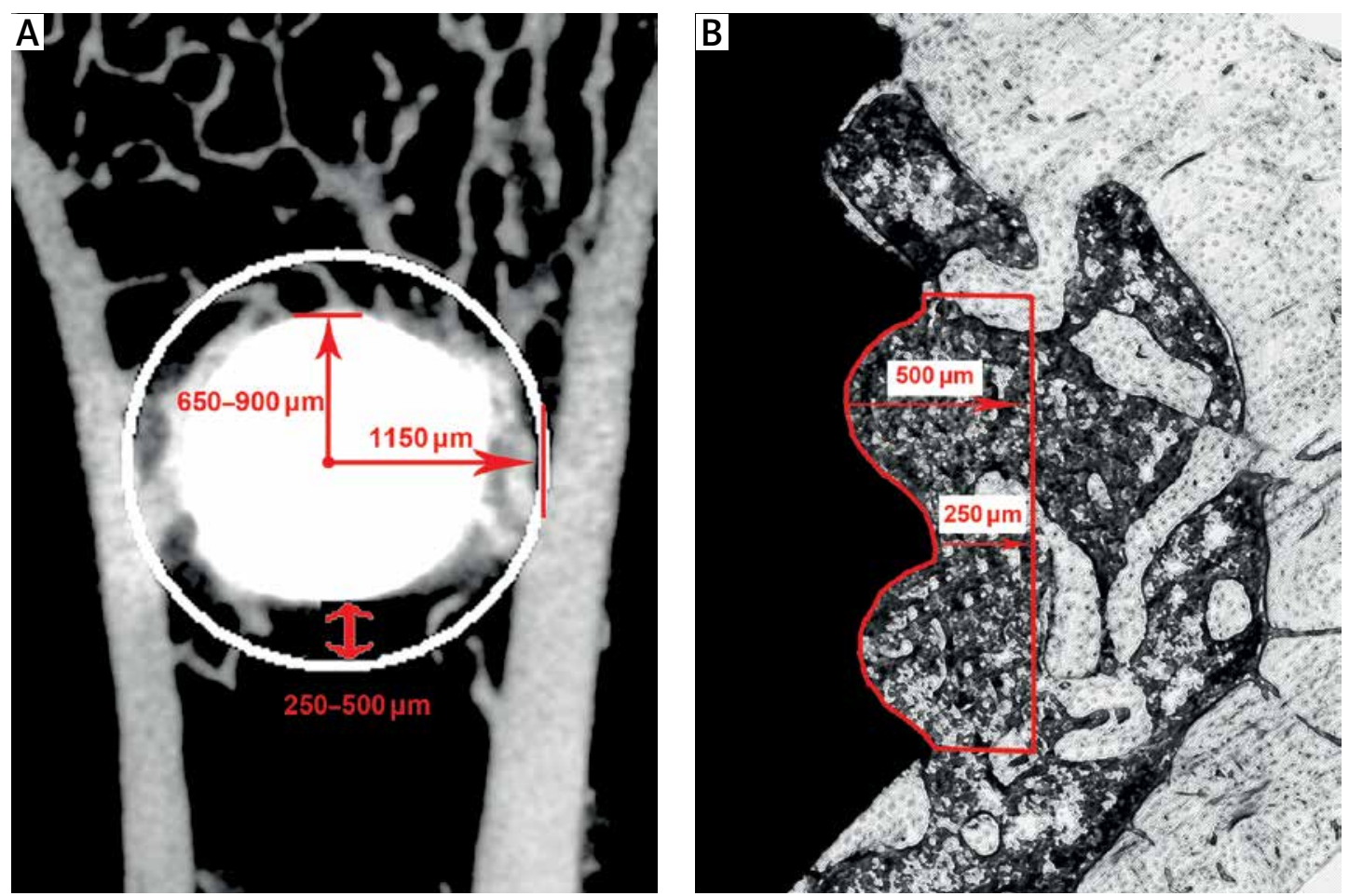

Figure 2. Scheme shows the volume of interest (VOI) in $\mu \mathrm{CT}$ analysis and region of interest (ROI) in histological evaluation. A - The VOI was defined as the trabecular compartment extending $1150 \mu \mathrm{m}$ from the longitudinal axis of the screw, namely 250-500 $\mu \mathrm{m}$ from the surface of screws, B - the ROI was similar to VOI but restricted to the area of the two front most threads 
4302; Instron, Norwood, MA, USA) using push-out force immediately after harvest. The specimens were prepared and the screws were pushed until out of the bone tissue as previously described [20, $21,23]$. The maximum force was calculated from the load-implant displacement data recorded.

\section{Statistical analysis}

Data are expressed as mean \pm standard deviation (SD). Statistical analyses were performed using the statistics package SPSS 17.0 (SPSS, Chicago, IL, USA). Comparison between groups was carried out using Student's t-test. A $p$-value below 0.05 was set as the significance level.

\section{Results}

\section{$\mu C T$ analysis}

In 3D and transverse 2D $\mu \mathrm{CT}$ images, the $\mathrm{OPG}$ group showed more cancellous bone around screws than the control (Figure 3). The quantitative results about peri-implant trabeculae and implant osseointegration are also presented in Figure 3. Compared to the control, OPG increased BV/ TV by $124 \%$, $\%$ OI by $167 \%$, Tb.N by $111 \%$, Tb.Th by $92 \%(p<0.01)$, and Conn.D by $95 \%(p<0.05)$; in contrast, Tb.Sp was decreased by $64 \%(p<0.05)$.

\section{Histological evaluation}

Histological images from control and OPG groups are exhibited in Figure 4. In quantitative analysis, OPG increased the BA by $160 \%$ and the BIC by $234 \%$ compared to the control $(p<0.01$; Figures 5 A, B).

\section{Biomechanical test}

The results of the biomechanical test are shown in Figure $5 \mathrm{C}$. When compared to the control, OPG increased the maximal push-out force by $228 \%$ $(p<0.01)$.

\section{Discussion}

Bone remodeling requires a precise balance between resorption and formation. The OPG/RANK/ RANKL signaling is the key pathway to regulation of the balance between bone resorption and formation. RANKL binds to its receptor RANK in order to induce osteoclast differentiation, activation, and function, whereas OPG acts as a decoy receptor to RANKL and therefore inhibits osteoclast activation and bone resorption [24, 25]. A normal ratio of OPG/RANKL, expected in homeostasis, will cause neither bone formation nor loss. Increased bone resorption, occurring in osteoporosis, is expected to be the result of an increase of RANKL relative to OPG.
With the increasing aging of the population, more and more people are affected by osteoporosis worldwide [2, 3, 26]. In order to explore the role of inhibition of RANKL on implant osseointegration, the effect of OPG, a decoy receptor as well as endogenous inhibitor of RANKL, was investigated using OVX rats in this study. Results from $\mu \mathrm{CT}$, histological, and biomechanical tests in our study in dicated that inhibition of RANKL by OPG increased peri-implant bone density, improved peri-implant trabecular microarchitecture, and enhanced implant fixation in OVX rats. It was suggested that OPG promoted implant osseointegration in osteoporotic bone. This positive effect of OPG on implant osseointegration in OVX rats should result from inhibited osteoclastic bone resorption due to inhibition of RANKL, although it was not confirmed in this study.

Previous animal studies have indicated the bone protective role of OPG, but this bone protective role might not be induced by promoting bone formation to a normal level or improving bone microarchitecture, but inhibiting bone resorption and increasing percent mineral composition [22, 27, 28]. Results from a fracture healing study in intact rat showed that OPG treatment did not influence the early callus expansion and fracture strength, but impaired the normal remodeling and consolidation processes during the subsequent period of remodeling without influencing structural fracture strength [29]. A different mechanism of bone fracture healing and bone healing around the implant might be the reason for different effects of OPG on fracture healing and implant fixation. It was important to increase callus formation at the early stage, as well as to maintain normal bone remodeling at the late stage of fracture healing. Thus, OPG did not work very well because it was not effective to increase callus formation but delayed callus remodeling. However, inhibition of excessive bone resorption around the implant, especially in osteoporotic bone, was very important for the initial stabilization of and long-term success of the implant [5].

Bernhardsson et al. recently found that 4-week treatment with osteoprotegerin with an Fc tag (OPG-Fc; $8 \mathrm{mg} / \mathrm{kg}$, twice weekly) in creased the pull-out force compared to saline controls by $153 \%$ ( $p<0.001)$. The OPG-Fc also increased the BV/TV in the previous drill hole and the bone density of the L5 vertebral body compared to the control [30]. This result was similar to the findings of this study but with a smaller effect (OPG increased the maximal push-out force by $228 \%$ in this study). Three plausible factors might contribute to the stronger effect of OPG on implant fixation in this study. First, a longer observation time was ap 

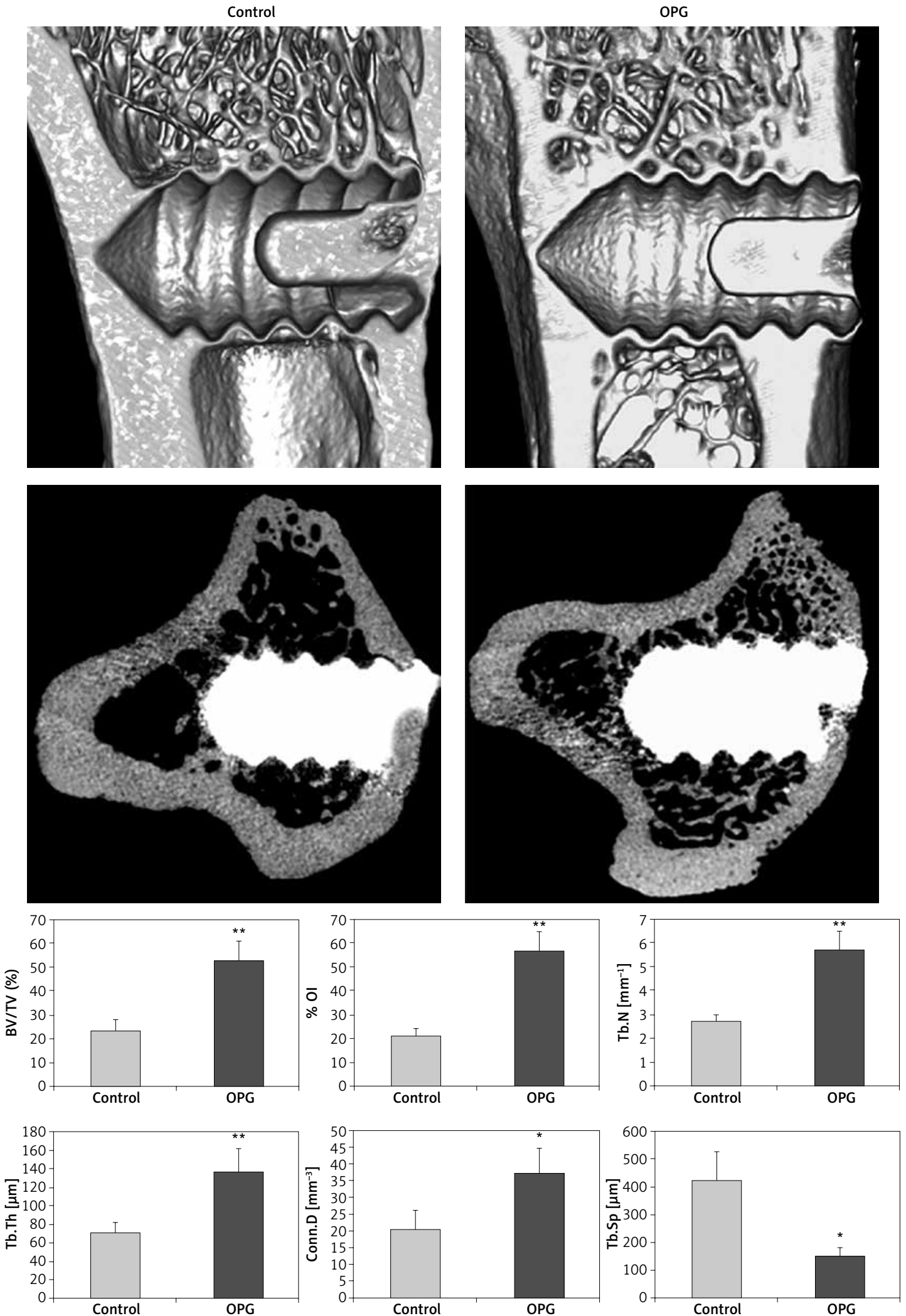

Figure 3. The 3D $\mu \mathrm{CT}$ images along the coronal plane of the tibiae (left, the upper row) and 2D transverse slices (left, the lower row) through the central part of the long axis of screws. The OPG group showed more cancellous bone around screws than control after 8-week treatment. In quantitative analysis (right), OPG significantly increased percent bone density and improved trabecular microstructure

BVITV - percent bone volume, \%OI - percent osseointegration, Conn.D - mean connective density, Tb.Th - mean trabecular thickness, Tb.N - mean trabecular number, Tb.Sp - mean trabecular separation. Data are expressed as mean \pm SD, error bars in the figure are presented as SD, $n=10$ specimens per group, ${ }^{*} p<0.05$ and ${ }^{* *} p<0.01 \mathrm{vs}$. control (Student's t-test) 

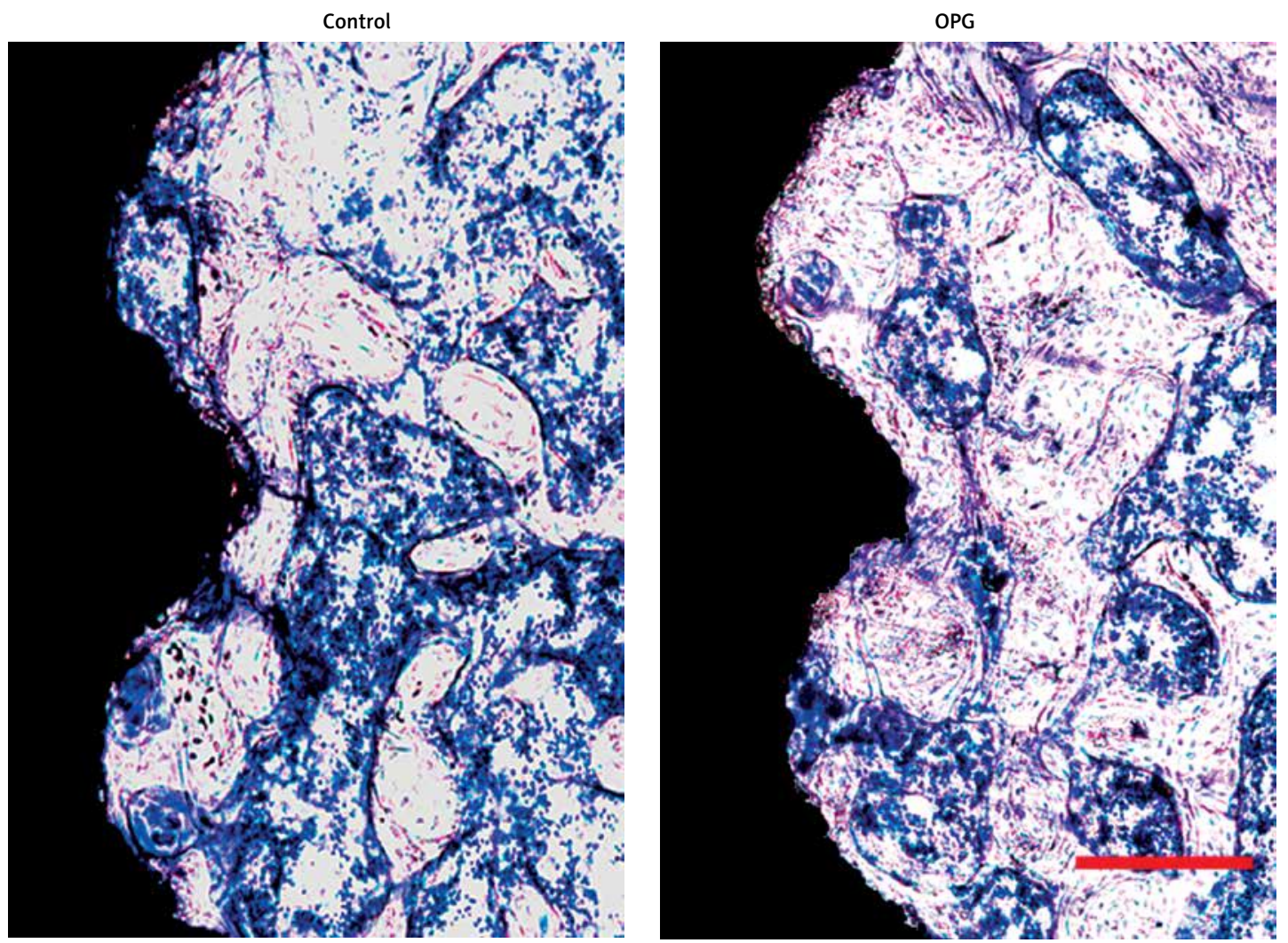

Figure 4. Histological images along the transverse plane of tibiae and through the central part of screws; toluidine blue stain, original magnification 100x, the scale bars represent $250 \mu \mathrm{m}$. The OPG group showed greater promotion of bone healing around screws than control after 8-week treatment

A

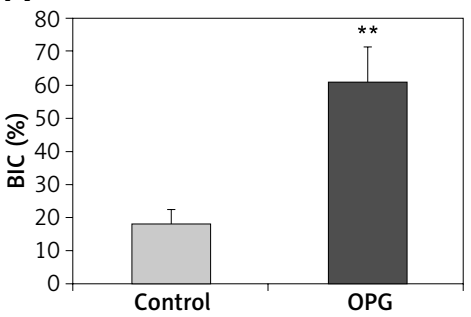

B

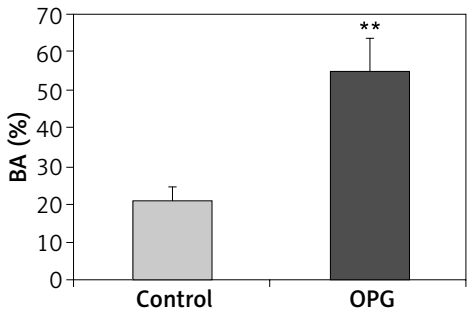

C

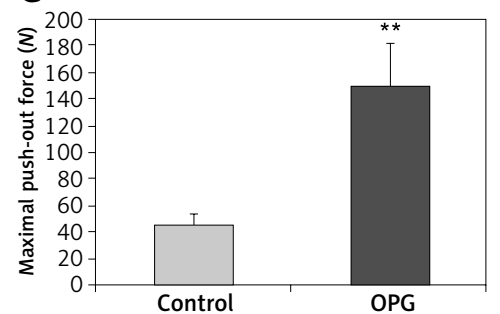

Figure 5. Quantitative results of histological evaluation (A, B) and biomechanical test (C) after 8-week treatment with OPG or vehicle. Compared to control, OPG significantly increased bone area density (BA), bone-to-implant contact (BIC), and the maximal push-out force of screws. Data are expressed as mean $\pm S D$, error bars in the figure are presented as SD, $n=10$ specimens per group, ${ }^{* *} p<0.01 \mathrm{vs}$. control (Student's $t$-test)

plied (8 weeks vs. 4 weeks); second, a higher dose of OPG treatment was used $(10 \mathrm{mg} / \mathrm{kg}$ three times a week vs. $8 \mathrm{mg} / \mathrm{kg}$ twice a week); third, a different animal model was used. The OVX rat used in this study was a classical animal model of post-menopausal osteoporosis due to estrogen deficiency and subsequently increased bone resorption. According to the mechanism of the bone protective role of OPG, it might work more effectively in OVX rats than intact rats [31].

In conclusion, the administration of OPG improved titanium screw fixation in OVX rats after 8 -week treatment. The enhancement of implant fixation was related to the increase of peri-implant bone density, improved trabecular microarchitecture, and enhanced implant osseointegration.

\section{Acknowledgments}

This study was supported by a grant from the National Natural Science Foundation of China (No. 81300858) and a grant from the National Basic Research Program of China (973 Program, No. 2012CB933902).

\section{Conflict of interest}

The authors declare no conflict of interest.

\section{References}

1. NIH Consensus Development Panel on Osteoporosis Prevention, Diagnosis, and Therapy. Osteoporosis prevention, diagnosis, and therapy. JAMA 2001; 285: 785-95. 
2. Liu Z, Piao J, Pang L, et al. The diagnostic criteria for primary osteoporosis and the incidence of osteoporosis in China. J Bone Miner Metab 2002; 20: 181-9.

3. Melton LJ 3rd, Chrischilles EA, Cooper C, Lane AW, Riggs BL. Perspective. How many women have osteoporosis? J Bone Miner Res 1992; 7: 1005-10.

4. Borsari V, Fini M, Giavaresi G, et al. Osteointegration of titanium and hydroxyapatite rough surfaces in healthy and compromised cortical and trabecular bone: in vivo comparative study on young, aged, and estrogen-deficient sheep. J Orthop Res 2007; 25: 1250-60.

5. Yamazaki $M$, Shirota T, Tokugawa Y, et al. Bone reactions to titanium screw implants in ovariectomized animals. Oral Surg Oral Med Oral Pathol Oral Radiol Endod 1999; 87: 411-8.

6. Shibli JA, Aguiar KC, Melo L, et al. Histological comparison between implants retrieved from patients with and without osteoporosis. Int J Oral Maxillofac Surg 2008; 37: 321-7.

7. Holahan CM, Koka S, Kennel KA, et al. Effect of osteoporotic status on the survival of titanium dental implants. Int J Oral Maxillofac Implants 2008; 23: 905-10.

8. Tsolaki IN, Madianos PN, Vrotsos JA. Outcomes of dental implants in osteoporotic patients. A literature review. J Prosthodont 2009; 18: 309-23.

9. Russell LA. Osteoporosis and orthopedic surgery: effect of bone health on total joint arthroplasty outcome. Curr Rheumatol Rep 2013; 15: 371.

10. Liu XL, Li CL, Lu WW, Cai WX, Zheng LW. Skeletal site-specific response to ovariectomy in a rat model: change in bone density and microarchitecture. Clin Oral Implants Res 2015; 26: 392-8.

11. Jacobs R, Ghyselen J, Koninckx P, van Steenberghe D. Long-term bone mass evaluation of mandible and lumbar spine in a group of women receiving hormone replacement therapy. Eur J Oral Sci 1996; 104: 10-6.

12. Yoshinari M, Oda Y, Inoue T, Matsuzaka K, Shimono $M$. Bone response to calcium phosphate-coated and bisphosphonate-immobilized titanium implants. Biomaterials 2002; 23: 2879-85.

13. Hilding M, Aspenberg P. Local preoperative treatment with a bisphosphonate improves the fixation of total knee prostheses: a randomized, double-blind radiostereometric study of 50 patients. Acta Orthop 2007; 78: 795-9.

14. Johansson HR, Skripitz R, Aspenberg P. Bisphosphonates can block the deterioration in implant fixation after withdrawal of intermittent doses of parathyroid hormone. J Bone Joint Surg Br 2008; 90: 400-4.

15. Nakashima T, Takayanagi $H$. New regulation mechanisms of osteoclast differentiation. Ann N Y Acad Sci 2011; 1240: E13-8.

16. Zebaze RM, Libanati C, Austin M, et al. Differing effects of denosumab and alendronate on cortical and trabecular bone. Bone 2014; 59: 173-9.

17. Tarantino U, Celi M, Feola M, Liuni FM, Resmini G, Iolascon G. A new antiresorptive approach to the treatment of fragility fractures: long-term efficacy and safety of denosumab. Aging Clin Exp Res 2013; 25 Suppl. 1: S65-9.

18. Kostenuik PJ, Nguyen HQ, McCabe J, et al. Denosumab, a fully human monoclonal antibody to RANKL, inhibits bone resorption and increases BMD in knock-in mice that express chimeric (murine/human) RANKL. J Bone Miner Res 2009; 24: 182-95.

19. Kostenuik PJ, Shalhoub V. Osteoprotegerin: a physiological and pharmacological inhibitor of bone resorption. Curr Pharm Des 2001; 7: 613-35.
20. Zhou C, Li Y, Wang X, Shui X, Hu J. 1,25Dihydroxy vitamin $D(3)$ improves titanium implant osseointegration in osteoporotic rats. Oral Surg Oral Med Oral Pathol Oral Radiol 2012; 114 (5 Suppl.): S174-8.

21. Li Y, Gao Y, Song G, Liu X, Hu J. Additive effects of estrogen replacement therapy and bisphosphonates on osseointegration of hydroxyapatite-coated titanium screws in ovariectomized rats. Oral Surg Oral Med Oral Pathol Oral Radiol Endod 2010; 109: 700-5.

22. Valenta A, Roschger P, Fratzl-Zelman N, et al. Combined treatment with PTH (1-34) and OPG increases bone volume and uniformity of mineralization in aged ovariectomized rats. Bone 2005; 37: 87-95.

23. Li Y, Feng G, Gao Y, Luo E, Liu X, Hu J. Strontium ranelate treatment enhances hydroxyapatite-coated titanium screws fixation in osteoporotic rats. J Orthop Res 2010; 28: 578-82

24. Trouvin AP, Goëb V. Receptor activator of nuclear factor-kappaB ligand and osteoprotegerin: maintaining the balance to prevent bone loss. Clin Interv Aging 2010; 5: 345-54.

25. Liu C, Walter TS, Huang P, et al. Structural and functional insights of RANKL-RANK interaction and signaling. J Immunol 2010; 184: 6910-9.

26. Burge R, Dawson-Hughes B, Solomon DH, Wong JB, King $A$, Tosteson $A$. Incidence and economic burden of osteoporosis-related fractures in the United States, 2005-2025. J Bone Miner Res 2007; 22: 465-75.

27. Ulrich-Vinther M, Andreassen TT. Osteoprotegerin treatment impairs remodeling and apparent material properties of callus tissue without influencing structural fracture strength. Calcif Tissue Int 2005; 76: 280-6.

28. Ulrich-Vinther M, Schwarz EM, Pedersen FS, Søballe K, Andreassen TT. Gene therapy with human osteoprotegerin decreases callus remodeling with limited effects on biomechanical properties. Bone 2005; 37: 751-8.

29. Gerstenfeld LC, Sacks DJ, Pelis M, et al. Comparison of effects of the bisphosphonate alendronate versus the RANKL inhibitor denosumab on murine fracture healing. J Bone Miner Res 2009; 24: 196-208.

30. Bernhardsson M, Sandberg O, Aspenberg P. Anti-RANKL treatment improves screw fixation in cancellous bone in rats. Injury 2015; 46: 990-5.

31. Liu F, Zhu J, Huang Y, et al. Contrast imaging and gene delivery through the combined use of novel cationic liposomal microbubbles and ultrasound in rat carotid arteries. Arch Med Sci 2013; 9: 347-53. 